\title{
ANÁLISE DE ALTERNATIVAS PRÁTICAS PARA REDUÇ̃̃o DE CUSTOS NA IMPLANTAÇÃO DE SISTEMAS DE CHUVEIROS AUTOMÁTICOS
}

\author{
ANALYSIS OF PRACTICAL ALTERNATIVES FOR REDUCING COSTS IN THE \\ IMPLEMENTATION OF SPRINKLERS
}

\author{
Ivan Ricardo Fernandes ${ }^{1}$ \\ Pablo Ruan Zotti Brum 2
}

Kelvin Gütz Marques 3

\section{Resumo}

Este artigo trata da análise de alternativas para a implantação de sistemas de chuveiros automáticos com menor custo. Foi analisada a utilização de distâncias entre bicos de sprinklers de até 3 metros ou mais, utilização de bucha ou luva de redução e o emprego de tubulações de aço carbono com ou sem galvanização, para regiões não litorâneas e não enterradas. Concluiuse, entre as alternativas citadas, quais apresentam um melhor custo benefício, para a implantação do sistema de sprinkler. Com a análise das pesquisas de viabilidade e de custo, houve uma notável vantagem no uso de distância entre bicos de até 3 metros, com redução no custo do material de $7,5 \%$ e redução no tempo de $20,9 \%$ a $11,8 \%$; foi determinado que a melhor alternativa para redução nos diâmetros de tubos é a bucha de redução, impactando em menor custo de material e tempo de instalação; e optou-se pelo uso de tubulação sem galvanização por representar uma considerável diferença no custo do material, entorno de $20 \%$.

Palavras-chave: Sprinklers. Otimização. Custo.

\begin{abstract}
This scientific article is about the analysis of alternatives for the implantation of automatic sprinklers systems with lower cost. It was analyzed the utilization of distances between sprinklers with 3 meters or more, the usage of "bucha" or "luva" of reduction and the use of carbon steel pipes with or without galvanization for non-coastal regions and not buried. At the end of the research, one of the alternatives presented a better cost benefit, for implementation of sprinkler systems. With the analyse of the research about feasibility and cost, it had a notable advantage in the use of distance between pipes up to 3 meters, with reduction in material cost of $7.5 \%$ and reduction in time from $20.9 \%$ to $11.8 \%$; It was determined that the best alternative for reduction in the pipe diameter is the "bucha" of reduction, impacting on lower material cost and shorter installation time. The use of non-galvanizing tubing was chosen because it represents a considerable difference in the cost of the material, of approximately $20 \%$.
\end{abstract}

Key words: Sprinklers. Optimization. Cost.

\footnotetext{
${ }^{1}$ Pontifícia Universidade Católica do Paraná, Mestre em Engenharia de Construção Civil, ivan.r@pucpr.br

${ }^{2}$ Pontifícia Universidade Católica do Paraná, Especialista em Engenharia de Segurança Contra Incêndio e Pânico, pabloruan.brum@gmail.com

${ }^{3}$ Serviço Nacional de Aprendizagem Industrial do Paraná, Técnico Mecânico, kelvingutz@hotmail.com
} 


\section{INTRODUÇ̃̃̃O}

A importância de medidas preventivas, quando o assunto é segurança, já é unanimidade na opinião de autoridades no assunto. Recentes tragédias envolvendo incêndios evidenciam a necessidade de medidas mais eficazes. Uma das melhores soluções, atualmente, para o combate de incêndio são os sistemas de sprinklers, os mesmos, além de serem automáticos, tem a capacidade, constatada em pesquisas internacionais, de controlar até $92 \%$ dos incêndios com a ativação de até 5 sprinklers (SKOP, 2017).

Apesar da eficiência em minimizar possíveis perdas, este sistema possuí um alto custo de implantação, dificultando a difusão dos sprinklers em território brasileiro. Desta forma torna-se extremamente importante o estudo referente a esse assunto, visando otimizações, permitidas pelas normas vigentes, que ocasionem em um custo menor desse sistema.

Sendo assim, o objetivo geral deste estudo é definir a melhor solução em relação ao custo benefício entre as opções apresentadas para a implantação de sistemas de chuveiros automáticos, analisando quais são as alternativas mais viáveis. Para o estudo será considerada a utilização de uma distância entre bicos maior que 3 metros ou até 3 metros; a utilização de componentes (luva de redução ou bucha de redução) para a redução de diâmetro em tubulações de até $50 \mathrm{~mm}$ (2"); e o tipo de tubulação (aço carbono sem galvanização ou aço carbono com galvanização) para regiões não litorâneas e não enterradas.

\section{DESENVOLVIMENTO}

\section{Distâncias de 3 metros entre bicos}

A primeira alternativa a ser abordada é quanto ao distanciamento entre bicos. Para realização dos estudos, foram geradas duas situações hipotéticas, sendo um 
Revista Científica do Corpo de Bombeiros Militar de Pernambuco

XVIII Seminário Nacional de Bombeiros - Foz do Iguaçu PR

Vol.04 No11 - Edição Especial XVIII SENABOM - ISSN 2359-4829

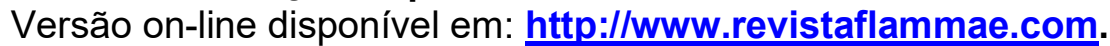

estabelecimento de $2.892,56 \mathrm{~m}^{2}$ para os dois casos, apresentando as mesmas características e parâmetros, sendo ambos enquadrados na classe de risco, contando apenas com diferença na disposição dos bicos, porém buscou-se que os bicos nas duas situações tivessem a mesma área de cobertura.

No caso 01 , a área de cobertura dos bicos é igual a $11,97 \mathrm{~m}^{2}$ e $12,00 \mathrm{~m}^{2}$ no caso 02. Vale mencionar que o caso 01 possui uma distância entre bicos $\mathrm{S}$ (distância entre bicos, conforme a ABNT NBR 10.897:2014) de 3,46 m e uma distância L (distância entre ramais, conforme a ABNT NBR 10.897:2014) de 3,46 m. Já no caso 02 temos a medida $\mathrm{S}$ igual a $3,00 \mathrm{~m}$ e $\mathrm{L}$ a $4,00 \mathrm{~m}$.

Como a dimensão $\mathrm{S}$ diminuiu do caso 01 para o caso 02 , houve a necessidade de aumentar a quantidades de bicos nesse ramal, entretanto a distância L aumentou, diminuindo a quantidades necessárias de ramais. Dessa maneira, no caso 01 se fez necessários 242 bicos e no caso 02 foram 247 pontos de chuveiros automáticos para a proteção adequada do suposto estabelecimento.

De acordo com a NBR 10.897:2014 há uma especificação referente às distâncias máximas e mínimas entre bicos, sendo respectivamente $4,60 \mathrm{~m}$ e $1,80 \mathrm{~m}$, além da sua área de cobertura máxima, de $12,1 \mathrm{~m}^{2}$. Desta forma a distância de 3 metros entre chuveiros automáticos, está em um intervalo possível e amparado pela norma regulamentadora.

Os impactos gerados por esta alternativa de distanciamento são diversos, englobando materiais, o tempo de mão-de-obra requisitada e a seleção da bomba. Para quantificar os impactos gerados em comparação ao caso 01 , anteriormente citado, foram seguidos alguns procedimentos, sendo: levantamento de materiais, estudo de tempo de mão-de-obra e cálculos para o dimensionamento das bombas.

Com a lista de materiais para fabricação elaborada para ambos os casos, obteve-se o custo de $\mathrm{R} \$ 41.300,95$, para o caso 01 e $\mathrm{R} \$ 38.204,58$ para o caso 02 , envolvendo o custo médio dos materiais requisitados. Logo, o caso 02 apresenta uma economia de 7,5\%, em relação ao caso 01 . Vale ressaltar que, como o objetivo é descobrir a diferença de um projeto para o outro, não foi levantado os materiais 
referente à casa de bombas e coluna principal, visto que serão iguais nos dois projetos. Ou seja, as conexões e válvulas nesse trecho serão iguais, entretanto, pode existir diferença na bomba selecionada. $\mathrm{O}$ dimensionamento da bomba será demonstrado ao decorrer deste artigo.

Para estipular os impactos gerados na mão-de-obra necessária nos dois casos apresentados, se fez necessário mapear os processos globais para execução dos dois sistemas, possibilitando então a constatação de quais procedimentos serão distintos ou apresentarão tomadas de tempo diferentes. Vale ressaltar que não está sendo considerado o tempo necessário para a casa de bombas e nem para a coluna principal, como já explicado anteriormente.

Com o mapeamento de ambos os casos definidos, algumas observações foram consideradas, entre elas a de que os processos de pintura e de fabricação e instalação de suportes serão distintos. Para a pintura, basta ser estimado através da metragem e diâmetros dos tubos. Já para a fabricação e instalação dos suportes, os mesmos foram classificados por unidade, não distinguindo o diâmetro da tubulação que irá alimentar, já que ao se alterar o diâmetro do tubo mudará somente o tipo do suporte e o mesmo já vem pronto, assim o tempo para um suporte de 4" acaba sendo igual ao suporte de 1".

Para o processo de fabricação e instalação de peças soldadas, o mesmo foi determinado conforme a quantidade de peças, e segundo especialistas tem-se diferença no tempo somente entre peças grandes e peças pequenas. Desta forma, tem-se 22 peças pequenas soldadas no caso 01,20 peças pequenas soldadas no caso 02,21 peças grandes soldadas no caso 01 e 18 peças grandes soldados no caso 02 . Logo, para esse processo pode-se concluir que se gasta $10 \%$ de tempo a mais no caso 01 para as peças soldadas pequenas e aproximadamente $15 \%$ de tempo a mais no caso 01 . Com isso não será levantado o tempo por operação para esse processo.

Outras etapas como teste de estanqueidade e mobilização das equipes para execução dos serviços, foram desconsiderados, uma vez que os tempos requisitados são praticamente iguais em ambos os casos. 
Revista Científica do Corpo de Bombeiros Militar de Pernambuco

XVIII Seminário Nacional de Bombeiros - Foz do Iguaçu PR

Vol.04 No11 - Edição Especial XVIII SENABOM - ISSN 2359-4829

Versão on-line disponível em: http://www.revistaflammae.com.

Com as observações feitas acima, a tabela 1 relaciona o tempo consumido para executar a pintura, sendo o tempo por metro para cada barra, e ainda o tempo para fabricação e instalação dos suportes. Ainda houve um acréscimo de $10 \%$ no tempo de final do processo de pintura, pois a cronometragem ocorreu em oficina e com boas condições de serviço.

Tabela 1 - Tomada de tempo para pintura e suportes

\begin{tabular}{|l|c|c|c|}
\hline DESCRIÇÃO MATERIAL & TEMPO (s) & $\begin{array}{c}\text { PEÇAS } \\
\text { CASO 01 }\end{array}$ & $\begin{array}{c}\text { PEÇAS } \\
\text { CASO 02 }\end{array}$ \\
\hline PINTURA - TUBO NBR 5580, CLASSE MÉDIA, Ø 1" & 490 & 26 & 19 \\
\hline PINTURA - TUBO NBR 5580, CLASSE MÉDIA, Ø 1.1/4" & 627 & 26 & 19 \\
\hline PINTURA - TUBO NBR 5580, CLASSE MÉDIA, Ø 1.1/2" & 784 & 51 & 38 \\
\hline PINTURA - TUBO NBR 5580, CLASSE MÉDIA, Ø 2" & 980 & 19 & 25 \\
\hline PINTURA - TUBO NBR 5580, CLASSE MÉDIA, Ø 2.1/2" & 1235 & 2 & 2 \\
\hline PINTURA - TUBO NBR 5580, CLASSE MÉDIA, Ø 3" & 1568 & 1 & 1 \\
\hline PINTURA - TUBO NBR 5580, CLASSE MÉDIA, Ø 4" & 1960 & 12 & 11 \\
\hline $\begin{array}{l}\text { FABRICAÇÃO E INSTALAÇÃO DE SUPORTE TIPO GOTA, } \\
\text { COM 0,3M DE BARRA ROSQUEADA }\end{array}$ & 360 & 264 & 228 \\
\hline
\end{tabular}

Fonte: Os autores (2018)

Para o processo de fabricação e instalação de peças rosqueadas será estudado por operações. Sendo separados da seguinte maneira: o tempo para se cortar uma barra no tamanho da medida da peça certa e abrir rosca nas duas extremidades do tubo; o tempo para passar o material vedante nas duas roscas e apertar a conexão em uma das extremidades; o tempo para instalar a peça no suporte e apertar na peça instalada anteriormente.

Com as tomadas de tempo realizadas e com a lista de materiais necessários para ambos os casos, ainda foi dado um acréscimo de $20 \%$ nos tempos, já que esse processo não será estudado no nível que ficará o sistema de sprinklers, ou seja, em um nível elevado. Com isso, o tempo final para todo processo, consiste na soma dos três sub processos, considerando as peças para cada caso.

Outro procedimento a ser analisado é referente à instalação dos bicos de sprinklers. Da mesma forma que os demais processos foram cronometrados os tempos para a instalação de bicos. Porém, como o mesmo é feito após o teste de estanqueidade 
Revista Científica do Corpo de Bombeiros Militar de Pernambuco

XVIII Seminário Nacional de Bombeiros - Foz do Iguaçu PR

Vol.04 No11 - Edição Especial XVIII SENABOM - ISSN 2359-4829

Versão on-line disponível em: http://www.revistaflammae.com.

foi considerado retirar o bujão de 1/2", preparar o bico e instalá-lo. Como as condições de instalação dos bicos serão em posição elevada, considerou-se também de $20 \%$ a mais no tempo marcado para condições perfeitas. Desta forma, segue a tabela 2 relacionando o tempo estimado aproximado para cada processo e os tempos totais aproximados para cada caso.

Tabela 2 - Comparativo de tomadas de tempos gerais

\begin{tabular}{|l|l|l|}
\hline DESCRIÇÃO DE PROCESSO & CASO 01 (s) & CASO 02 (s) \\
\hline CORTE E ROSQUEAMENTO & 147510 & 104994 \\
\hline PINTURA & 115209 & 101116 \\
\hline INSTALAÇÃO DE MATERIAL VEDANTE E APERTO DE UMA CONEXÕES & 69603 & 51710 \\
\hline FABRICAÇÃO E INSTALAÇÃO DE SUPORTES & 95040 & 82080 \\
\hline SUPORTE E APERTO DA PEÇA NA PEÇA ANTERIORMENTE INSTALADA & 69811 & 50403 \\
\hline INSTALAÇÃO DE BICOS & 26620 & 27170 \\
\hline TOTAL (SEGUNDOS) & 523793 & 417474 \\
\hline TOTAL (HORAS) & 145 & 116 \\
\hline
\end{tabular}

Fonte: Os autores (2018)

Baseado no estudo elaborado se faz possível analisar uma redução de 20,7\% no tempo requisitado para o tempo de mão-de-obra, em comparação ao caso 01. Mas vale a observação que a metodologia desenvolvida ocorreu em um ambiente com boas condições de trabalho e não mensuram todos os tempos como a organização das ferramentas, a seleção dos materiais, a conferência de medidas no projeto e demais serviços necessários e intrínsecos ao serviço como um todo, os quais poderiam vir a impactar nas conclusões obtidas.

Além de utilizar essa metodologia desenvolvida pelos autores, seguiu-se alguns parâmetros estabelecidos por Pedro Telles (2001), onde o mesmo desenvolveu uma tabela com tempo para estimativa de tempos de homem-hora para serviços de instalação de tubulações. Utilizando-se esses parâmetros foi possível desenvolver a tabela 3 para os casos estudados. 
Revista Científica do Corpo de Bombeiros Militar de Pernambuco

XVIII Seminário Nacional de Bombeiros - Foz do Iguaçu PR

Vol.04 No11 - Edição Especial XVIII SENABOM - ISSN 2359-4829

Versão on-line disponível em: http://www.revistaflammae.com.

Tabela 3 - Tabela homem-hora, conforme literatura

\begin{tabular}{|c|c|c|c|c|c|}
\hline \\
\hline \multicolumn{6}{|c|}{$\begin{array}{l}\text { ESTIMATIVA DE TEMPO DE MÃO-DE-OBRA - CASO } 01 \text { E } 02 \\
\begin{array}{|l|l|l|l|l|l|}\text { DESCRIÇÃO } & \text { UND } & \begin{array}{l}\text { QTDE } \\
\text { CASO 01 }\end{array} & \begin{array}{l}\text { HOMEM/HORA } \\
\text { CASO 01 }\end{array} & \begin{array}{l}\text { QTDE } \\
\text { CASO 02 }\end{array} & \begin{array}{l}\text { HOMEM/HORA } \\
\text { CASO 02 }\end{array} \\
\end{array}\end{array}$} \\
\hline \multicolumn{6}{|c|}{\begin{tabular}{l|l|l|l|l|l} 
TUBO NBR 5580, CLASSE MÉDIA, Ø 1" & m & 156 & 37,44 & 114
\end{tabular}} \\
\hline TUBO NBR 5580, CLASSE MÉDIA, Ø 1.1/4" & $\mathrm{m}$ & 156 & 40,56 & 114 & 29,64 \\
\hline TUBO NBR 5580, CLASSE MÉDIA, Ø 1.1/2" & $\mathrm{m}$ & 306 & 91,8 & 228 & 68,4 \\
\hline TUBO NBR 5580, CLASSE MÉDIA, Ø 2" & $\mathrm{m}$ & 114 & 44,46 & 150 & 58,5 \\
\hline CONEXÕES P/ FG BSP - Ø 2" & pç & 110 & 132 & 133 & 159,6 \\
\hline CONEXÕES P/ FG BSP - Ø 1.1/2" & pç & 169 & 135,2 & 114 & 91,2 \\
\hline CONEXÕES P/ FG BSP - Ø 1.1/4" & $\mathrm{pç}$ & 106 & 68,9 & 76 & 49,4 \\
\hline \multirow[t]{2}{*}{ CONEXÕES P/FG BSP - Ø 1" } & $\mathrm{pç}$ & 62 & 31 & 57 & 28,5 \\
\hline & & $\begin{array}{l}\text { TOTAL } \\
\text { CASO } 01\end{array}$ & 581,36 & \multicolumn{2}{|c|}{$\begin{array}{l}\text { TOTAL } \\
\text { CASO } 02\end{array}$} \\
\hline
\end{tabular}

Fonte: Os autores (2018)

O último ponto a se analisar nessa alternativa apresentada é o impacto gerado no dimensionamento na bomba. Baseado na NBR 10897:2014, seguiu-se a metodologia de cálculo para se dimensionar os dois sistemas e selecionar uma bomba para ambos os casos.

Como resultado, obteve-se a seleção da mesma bomba para os dois casos. Com apenas uma variação de $1 \%$ nos parâmetros para seleção da bomba, não influenciou na bomba selecionada, já que geralmente utiliza-se bombas com curva de funcionamento levemente acima da mínima necessária, assim utilizando bombas com rotor padrão.

\section{Bucha de redução $x$ Luva de redução}

A segunda alternativa a ser abordada é quanto ao uso de buchas e luvas de redução. Buscando encontrar o melhor custo x benefício, o estudo realizado comparou as peças citadas conforme viabilidade teórica e pratica, se as mesmas são recomendas por norma e se existe alguma restrição. Após isto uma análise em relação ao custo destas peças, em seguida as diferenças na mão de obra e por final se existe algum impacto na bomba, ao se optar por uma ou outra alternativa.

Visando a viabilidade da aplicação, a NBR10897:2014 aponta no item 5.5.3 que as duas peças devem ser aplicadas quando existir mudanças no diâmetro da 
Revista Científica do Corpo de Bombeiros Militar de Pernambuco

XVIII Seminário Nacional de Bombeiros - Foz do Iguaçu PR

Vol.04 No11 - Edição Especial XVIII SENABOM - ISSN 2359-4829

Versão on-line disponível em: http://www.revistaflammae.com.

tubulação. Logo, não há restrições normativas ou técnicas para o uso de luvas ou buchas de redução. Entretanto, ainda no mesmo item citado, a norma recomenda o uso da luva de redução.

Para confrontar os custos da utilização de bucha de redução contra luvas de redução, deve-se considerar o custo direto e indireto para a utilização do componente. Analisando a instalação da luva de redução, como possui duas roscas do tipo fêmea e a redução de diâmetro da tubulação é sempre feito após uma conexão que também possui rosca fêmea, sendo necessária uma peça com extremidades com rosca macho, para efetuar a união.

Sendo assim, em piores condições, se faz necessário o uso de um niple. Devendo esta peça ter seu custo acoplado ao custo de utilização de luvas de redução. Em contrapartida, ao utilizar-se do niple duplo, tem-se uma pequena diminuição no tamanho do tubo, com isso economiza-se com os tubos. Desta maneira, será confrontado o custo das buchas de redução contra o custo da luva de redução mais o niple e menos o pedaço de tubo que se deve diminuir da peça. As tabelas 04 e 05 relacionam os preços das peças e os seus custos indiretos.

Tabela 4 - Caso 3 - Custos de materiais - Luvas de redução

\begin{tabular}{|c|c|c|c|c|}
\hline DESCRIÇÃO & UND & QTDE & \multicolumn{2}{|c|}{ R\$ } \\
\hline LUVA DE REDUÇÃO GALVANIZADA, F/F, BSP - Ø 2" x 1.1/2" & pç & 1 & $\mathrm{R} \$$ & 30,45 \\
\hline LUVA DE REDUÇÃO GALVANIZADA, F/F, BSP - Ø 1.1/2" x 1.1/4" & pç & 1 & $\mathrm{R} \$$ & 25,98 \\
\hline LUVA DE REDUÇÃO GALVANIZADA, F/F, BSP - $\varnothing 1.1 / 4 "$ x $1 "$ & pç & 1 & $\mathrm{R} \$$ & 20,96 \\
\hline NIPLE DUPLO GALVANIZADO, BSP - Ø 2" & pç & 1 & $\mathrm{R} \$$ & 14,13 \\
\hline NIPLE DUPLO GALVANIZADO, BSP - Ø $1.1 / 2 "$ & pç & 1 & $\mathrm{R} \$$ & 10,33 \\
\hline NIPLE DUPLO GALVANIZADO, BSP - Ø 1.1/4" & pç & 1 & $\mathrm{R} \$$ & 7,43 \\
\hline TUBO NBR 5580, CLASSE MÉDIA, Ø 2" - 68mm (A DESCONTAR) & pç & 1 & $-\mathrm{R} \$$ & 2,57 \\
\hline TUBO NBR 5580, CLASSE MÉDIA, Ø 1.1/2" - 59mm (A DESCONTAR) & Pç & 1 & $-\mathrm{R} \$$ & 1,39 \\
\hline TUBO NBR 5580, CLASSE MÉDIA, Ø 1.1/4" - 57mm (A DESCONTAR) & Pç & 1 & $-\mathrm{R} \$$ & 1,19 \\
\hline
\end{tabular}

Fonte: Os autores (2018) 
Revista Científica do Corpo de Bombeiros Militar de Pernambuco

XVIII Seminário Nacional de Bombeiros - Foz do Iguaçu PR

Vol.04 No11 - Edição Especial XVIII SENABOM - ISSN 2359-4829

Versão on-line disponível em: http://www.revistaflammae.com.

Tabela 5 - Caso 3 - Custos de materiais - Buchas de redução

\begin{tabular}{|l|c|c|cc|}
\hline DESCRIÇÃO & UND & QTDE & \multicolumn{2}{|c|}{ R\$ } \\
\hline BUCHA DE REDUÇÃO GALVANIZADA, M/F, BSP - Ø 2" x 1.1/2" & Pç & 1 & R\$ & 11,77 \\
\hline BUCHA DE REDUÇÃO GALVANIZADA, M/F, BSP - Ø 1.1/2" x 1.1/4" & Pç & 1 & R\$ & 8,51 \\
\hline BUCHA DE REDUÇÃO GALVANIZADA, M/F, BSP - Ø 1.1/4" x 1" & Pç & 1 & R\$ & 7,24 \\
\hline \multicolumn{2}{|r|}{ TOTAL } & R\$ & $\mathbf{2 7 , 5 1}$ \\
\hline
\end{tabular}

Fonte: Os autores (2018)

Com isso percebe-se uma diferença considerável para o uso de luvas de redução, onde o emprego de buchas consiste em apenas $26,4 \%$ do custo das luvas.

Do mesmo modo apresentado na alternativa anterior, do distanciamento de 3,0 m entre bicos, será feito um estudo para concluir qual das alternativas demanda um maior tempo para a execução. E analisando novamente todos os processos que envolvem a instalação de sprinklers, percebe-se que a utilização de bucha ou luva de redução somente irá impactar no processo de aperto das conexões nas peças de tubos.

A tabela 6 evidencia resultados médios para tempos de aperto das conexões. O uso do tee (T), apresentado na tabela, não teve um motivo elementar, apenas foi necessário usar peças com rosca fêmea para apertar a bucha e luva de redução com o niple duplo.

Tabela 6 - Tomada de tempo - Processo: Bucha de redução x Luva de redução

\begin{tabular}{|l|c|c|}
\hline DESCRIÇÃO MATERIAL & $\begin{array}{c}\text { TEMPO (S) - PEÇAS } \\
\text { COM BUCHA }\end{array}$ & $\begin{array}{c}\text { TEMPO (S) - PEÇAS } \\
\text { COM LUVA E NIPLE }\end{array}$ \\
\hline TEE GALVANIZADO, F/F, BSP - Ø 1" & 192 & 253 \\
\hline TEE GALVANIZADO, F/F, BSP - Ø 1.1/4" & 325 & 345 \\
\hline TEE GALVANIZADO, F/F, BSP - Ø 1.1/2" & 301 & 332 \\
\hline TEE GALVANIZADO, F/F, BSP - Ø 2" & 311 & 355 \\
\hline TOTAL (SEGUNDOS) & 1129 & 1285 \\
\hline TOTAL (MIN) & 19 & 21 \\
\hline
\end{tabular}

Fonte: Os autores (2018)

Da análise da tabela, percebe-se que existe uma redução no tempo de mãode-obra de aproximadamente $9,5 \%$ ao utilizar buchas de redução se comparadas com as 
Revista Científica do Corpo de Bombeiros Militar de Pernambuco

XVIII Seminário Nacional de Bombeiros - Foz do Iguaçu PR

Vol.04 No11 - Edição Especial XVIII SENABOM - ISSN 2359-4829

Versão on-line disponível em: http://www.revistaflammae.com.

luvas, referente ao tempo para instalação e aperto dos componentes, ou seja, a bucha de redução possui um menor tempo para instalação.

Outro processo que também sofrerá um impacto indireto com a utilização de luva ou bucha de redução é o teste de estanqueidade, em que ocorrer vazamentos, e como para o emprego de luva de redução existirá uma maior quantidade de uniões entre rosca, a probabilidade de vazamentos aumenta, tomando mais tempo para a realização do teste de estanqueidade.

O último ponto a se analisar nesta alternativa é o impacto gerado na seleção da bomba. A principal diferença que se tem ao optar pela bucha ou luva de redução no cálculo e dimensionamento do sistema é a perda de carga localizada para cada componente. Os dois possuem uma pequena diferença de valores, conforme demonstrado na figura 1.

Figura 1 - Equivalência da perda de carga das conexões (luva e bucha de redução) em metros de tubos.

\begin{tabular}{|c|c|c|c|c|c|c|c|c|c|c|c|c|c|c|c|}
\hline \multirow{2}{*}{ 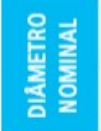 } & $1 / 2 \times 1 / 4$ & $1 / 2 \times 36$ & $x_{1} \times x_{4}$ & $3 / x<x$ & $3 / 4 \times 1 / 2$ & $1 \times 3 / 2$ & $1 \times 1 / 2$ & $1 x^{3 / 6}$ & $14 / 4 \times 1 / 2$ & $174 \times 3 \times 3$ & $18 / 4 \times 1$ & $112 \times 1 / 2$ & $172 \times 3 / 6$ & $1 / 2 \times 1$ & $11 / 2 \times 11 / 4$ \\
\hline & $2 x * \pi$ & $2 \times 1$ & $2 \times 1 \%$ & $2 \times 1 \%$ & $28 / 2 \times 1$ & $212 \times 1144$ & $21 / 2 \times 1 \%$ & $212 \times 2$ & $3 \times 1$ & $3 \times 1 \times 4$ & $3 \times 1 \%$ & $3 \times 2$ & $3 \times 2 / 2$ & $4 \times 2$ & $4 \times 3$ \\
\hline 0,10 & 0,16 & 0,14 & 0,22 & 0,23 & 0,24 & & 0,24 & 0,24 & 0,24 & 0,22 & 0,19 & 0,29 & 0,26 & 0,24 & 0,20 \\
\hline 0,34 & & 0,36 & 0,40 & 0,43 & 0,28 & 0,33 & 0,36 & 0,39 & & 0,65 & 0,69 & 0,75 & & & \\
\hline 0,11 & 0,18 & 0,18 & & 0,26 & 0,32 & 0,30 & 0,32 & 0,29 & 0,33 & 0,43 & 0,16 & & 0,53 & 0,27 & 0,12 \\
\hline & & 0,30 & 0,35 & 0,38 & & 0,44 & 0,48 & 0,64 & & & 0,71 & 0,70 & 0,71 & & \\
\hline
\end{tabular}

Fonte: TUPY (2009)

Realizando os procedimentos de cálculo presentes na norma, já citada na alternativa anterior, percebe-se que houve uma diferença mínima na altura manométrica e na vazão para dimensionamento da bomba na ordem $0,2 \%$, ou seja, impactando novamente na seleção da mesma bomba para as opções.

\section{Sistemas com e sem galvanização}


Revista Científica do Corpo de Bombeiros Militar de Pernambuco

XVIII Seminário Nacional de Bombeiros - Foz do Iguaçu PR

Vol.04 No11 - Edição Especial XVIII SENABOM - ISSN 2359-4829

Versão on-line disponível em: http://www.revistaflammae.com.

Nesta última alternativa o ambiente hipotético de aplicação será o de utilização de tubulação com galvanização ou não para sistemas em regiões não litorâneas e não enterradas. De semelhante modo as alternativas anteriores, serão analisados dois casos, sendo sistemas com mesma área e demais características, apenas diferenciando na especificação das tubulações. Em um dos casos se utilizará o aço carbono com tubulação não galvanizada e em outro aço carbono com tubulação galvanizada.

Objetivando facilitar todas as análises será utilizado o caso 02 , citado anteriormente, onde o mesmo será com tubulações sem galvanização e conexões rosqueadas até $50 \mathrm{~mm}$ (2") e soldadas para diâmetros maiores de $63 \mathrm{~mm}(2.1 / 2$ "). O caso 04 será semelhante, porem com tubulações com galvanização e conexões rosqueadas em todo o sistema.

Para início das análises, novamente constata-se a viabilidade da alternativa através das normas vigentes. A norma brasileira, a qual não apresenta nenhuma preferência, tubulações com ou sem galvanização, apenas aponta a norma que os tubos devem seguir, conforme a forma de junção, para tubos unidos por conexões soldadas deve-se atender a ABNT NBR 5580 (classe leve), no mínimo e para tubos unidos por conexões rosqueadas a ABNT NBR 5580 (classe média) no mínimo.

Porém, como o aço-carbono não é um material de alta resistência à corrosão, é gerada a necessidade de um acréscimo de espessura, criando uma margem para possíveis corrosões nas partes externas expostas ao contato com a atmosfera. (TELLES, 2001)

Desta maneira, adotou-se tubulações conforme a ABNT NBR 5580:2015 classe média (com parede de tubo mais espessa) para ambos os casos, pois para os tubos sem galvanização, haverá sobra de material para garantir a qualidade e proteção a corrosão, a qual já é prevista.

O processo de galvanização também pode impactar na forma de unir os tubos, uma vez que a soldagem em componentes de aço galvanizados a fogo pode necessitar de processos diferentes, comparados aos não revestidos. Isso se deve ao fato 
Revista Científica do Corpo de Bombeiros Militar de Pernambuco

XVIII Seminário Nacional de Bombeiros - Foz do Iguaçu PR

Vol.04 N011 - Edição Especial XVIII SENABOM - ISSN 2359-4829

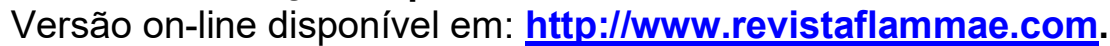

de que a soldagem gera uma perda de parte da camada de revestimento, já que a mesma tende a ser volatizada durante o procedimento. (PANONNI, 2015)

Com estas observações decidiu-se aplicar para os estudos do custo dos materiais, da mão-de-obra e dos impactos na seleção da bomba, dois sistemas semelhantes ao caso 02, da primeira alternativa apresentada neste artigo. A diferença será apenas para o caso estudado com as tubulações com galvanização, onde as conexões serão rosqueadas.

Desta maneira, o estudo baseou-se nas peças de fabricação e a lista de materiais necessária do caso 02 , apenas se alterou o tipo de tubulação, para tubulação com galvanização e as conexões soldadas foram convertidas para conexões rosqueadas, assim temos o caso 04 para análise.

Assim, para o caso 02, como já visto, se tem um custo para os materiais de $\mathrm{R} \$ 38.204,58$ e para o caso 04 o valor de $\mathrm{R} \$ 47.237,40$. Esclarecendo que o sistema com tubos de aço sem galvanização representa um custo de materiais de apenas $80,8 \%$ do custo de materiais para o sistema com tubos galvanizados.

Já para estudo das tomadas de tempo para quantificação do tempo requisitado nos dois casos, constatou-se que haverá diferença apenas nos processos para tubos acima de $65 \mathrm{~mm}$ (2.1/2"), pois os casos com tubulação inferiores a $65 \mathrm{~mm}$ (2.1/2") são exatamente iguais, já que os processos de corte, rosqueamento, aperto de peça, instalação de suportes e pintura, não sofrem alterações com a mudança na especificação dos tubos.

Sabendo disso, foi feita a cronometragem dos processos, de forma semelhante para se realizar o projeto, conforme o caso 02 e o caso 04 , porem em oficina e em condições boas de trabalho. Nas tabelas 7 e 8 seguem os tempos totais para os processos distintos dos casos 02 e 04 . 


\section{Revista FLAMMAE}

Revista Científica do Corpo de Bombeiros Militar de Pernambuco XVIII Seminário Nacional de Bombeiros - Foz do Iguaçu PR Vol.04 N¹1 - Edição Especial XVIII SENABOM - ISSN 2359-4829 Versão on-line disponível em: http://www.revistaflammae.com.

Tabela 7 - Tomada de tempos - Processo Caso 02

\begin{tabular}{|l|c|}
\hline DESCRIÇÃO DE PROCESSO & CASO 02 (s) \\
\hline CORTE E PREPARAÇÃO DO TUBO & 17481 \\
\hline PONTEAMENTO DA PEÇA COM A CONEXÃO E NIVELAMENTO & 15773 \\
\hline PROCESSO DE SOLDAGEM E ANÁLISE DA SOLDA & 207418 \\
\hline TOTAL (SEGUNDOS) & 240672 \\
\hline TOTAL (HORAS) & 67 \\
\hline
\end{tabular}

Fonte: Os autores (2018)

Tabela 8 - Tomada de tempos - Processo Caso 04

\begin{tabular}{|l|c|}
\hline DESCRIÇÃO DE PROCESSO & CASO 02 (s) \\
\hline CORTE E ROSQUEAMENTO & 54228 \\
\hline INSTALAÇÃO DE MATERIAL VEDANTE E APERTO DE UMA CONEXÕES & 24490 \\
\hline FABRICAÇÃO E INSTALAÇÃO DE SUPORTES & 25625 \\
\hline TOTAL (SEGUNDOS) & 104342 \\
\hline TOTAL (HORAS) & 29 \\
\hline
\end{tabular}

Fonte: Os autores (2018)

Desta forma percebe-se que os processos do caso 02 demandam muito mais tempo, devido ao processo de soldagem, o qual necessita de um controle maior de qualidade. Sendo que no caso 04 necessitou apenas de aproximadamente $43 \%$ do tempo do caso 02 .

Analisando conforme a metodologia de Telles (2001), temos as tabela 9 e 10.

Tabela 9 - Estimativa de homens/hora - caso 02

\begin{tabular}{|l|l|l|l|}
\hline CASO 2 - QTDE DE HOMENS/HORA P/ SERVIÇOS DE TUBULAÇÃO \\
\hline DESCRIÇÃO & UND & QTDE & Homem/Hora \\
\hline CONEXÕES P/ SOLDA SCH 40 - Ø 4" & pç & 20 & 50 \\
\hline CONEXÕES P/ SOLDA SCH 40 - Ø 3" & pç & 2 & 4 \\
\hline CONEXÕES P/ SOLDA SCH 40 - Ø 2.1/2" & pç & 53 & 90,1 \\
\hline & & TOTAL & $\mathbf{1 4 4 , 1}$ \\
\hline
\end{tabular}

Fonte: Os autores (2018)

Tabela 10 - Estimativa de homens/hora - caso 04

\begin{tabular}{|l|l|l|l|}
\hline CASO 4 - QTDE DE HOMENS/HORA P/ SERVIÇOS DE TUBULAÇ̃̃O \\
\hline DESCRIÇÃO & UND & QTDE & Homem/Hora \\
\hline CONEXÕES P/ FG BSP - Ø 4" & pç & 20 & 51,8 \\
\hline CONEXÕES P/ FG BSP - Ø 3" & pç & 2 & 4,04 \\
\hline CONEXÕES P/ FG BSP - Ø 2.1/2" & pç & 53 & 84,27 \\
\hline \multicolumn{4}{|c|}{ TOTAL $\mathbf{1 4 0 , 1 1}$} \\
\hline
\end{tabular}

Fonte: Os autores (2018) 
Quanto ao dimensionamento da bomba para os sistemas, trocar o material das tubulações e das conexões pode acarretar na perda de carga do sistema, devido a rugosidade e outros fatores dos materiais a serem diferentes, e esse dado é caracterizado pelo fator $\mathrm{C}$.

A tabela 25 da ABNT NBR 10.897:2014 apresenta os valores do fator C, conforme o material da tubulação. Para tubulações de aço carbono sem galvanização e com galvanização é utilizado o mesmo fator C.

Com isso nos cálculos para dimensionamento do sistema e para seleção da bomba não haverá diferenças, uma vez que se usa o mesmo fator $\mathrm{C}$. Sendo assim, não existem impactos na seleção da bomba e no dimensionamento do sistema.

\section{CONCLUSÕES}

Após todas as análises, consegue-se selecionar as melhores alternativas, ou seja, as que apresentam o melhor custo benefício.

Para o primeiro caso estudado, com a utilização de uma distância entre bicos de até 3 metros ou maior que 3 metros, ficou evidente que a alternativa com até 3 metros apresentou melhor desempenho, uma vez que resultou em uma quantidade menor de peças e menor quantidade de materiais, resultando em menor custo. Mesmo que para atender a alternativa seja necessário utilizar uma quantidade maior de bicos. Esse fato se deu, devido à utilização de tubos padrões, em que os mesmos são comercializados em barras de 6 metros, sendo que ao utilizar até 3 metros de distância entre bicos, é possível fazer duas peças com uma barra, sendo que com distâncias maiores, é necessário unir dois restos de barras para realizar a segunda peça.

Ainda nesse tópico, é possível estudar uma distância ideal entre bicos, onde a peça de fabricação já tem a medida de 3 metros, reduzindo ainda mais as perdas com sobras de materiais.

Para o segundo assunto estudado, a bucha de redução apresentou um melhor desempenho, uma vez que o componente tem um menor custo, e a sua utilização não 
Revista Científica do Corpo de Bombeiros Militar de Pernambuco

XVIII Seminário Nacional de Bombeiros - Foz do Iguaçu PR

Vol.04 No11 - Edição Especial XVIII SENABOM - ISSN 2359-4829

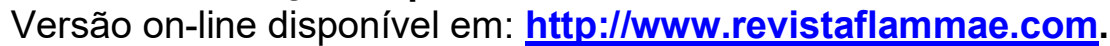

necessita de outras peças para complementar, além de facilitar o teste reduzindo pontos para possíveis vazamentos. Entretanto, existe uma recomendação na norma para o uso de luvas de redução, com isso incentiva-se a análise desse ponto de vista pela ABNT.

Para a última análise realizada, com a utilização de tubulação com ou sem galvanização, a alternativa sem galvanização apresentou um melhor desempenho. $\mathrm{O}$ valor do custo do material para o sistema sem galvanização teve uma grande diferença em detrimento ao com galvanização, entretanto nas estimativas dos tempos houve um impasse, já que pela literatura o tempo foi quase o mesmo, porém na cronometragem houve uma grande diferença.

Esta diferença se deve pelos ensaios serem realizados em bancada e oficina, e não se possível aferir o tempo total do processo, além de sofrer muita variação conforme a habilidade do soldador. Como existem recomendações contra o uso de peças galvanizadas para diâmetros maiores e iguais a $65 \mathrm{~mm}(2.1 / 2$ "), a alternativa utilizando tubulação sem galvanização se mostrou com maior vantagem.

Ao se comparar a diferença de tempo dado pela metodologia da cronometragem, visto que está se analisando somente os processos distintos entre os casos, a diferença de tempo encontrada represente somente $4 \%$ do tempo estimado total para a realização de todo serviço, ou seja, na pior das situações haverá uma diferença de apenas $4 \%$ no tempo total da obra. Vale citar que o ideal seria a cronometragem dos serviços em loco, assim buscando tempos mais próximos da realidade.

\section{REFERÊNCIAS}

ASSOCIAÇÃO BRASILEIRA DE NORMAS TÉCNICAS. NBR 10897: Sistemas de Chuveiros Automáticos para Combate a Incêndio, Rio de Janeiro, 2014.

BRENTANO, T. Instalações Hidráulicas de Combate a Incêndio nas Edificações. 4. Ed. Porto Alegre, 2011.

BRENTANO, T. Proteção Contra Incêndios no Projeto de Edificações. 1. Ed. Porto Alegre, 2007. 
Revista Científica do Corpo de Bombeiros Militar de Pernambuco XVIII Seminário Nacional de Bombeiros - Foz do Iguaçu PR

Vol.04 N011 - Edição Especial XVIII SENABOM - ISSN 2359-4829

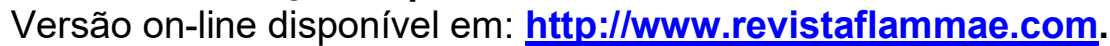

FERNANDES R. I. Sistema de Chuveiros Automáticos NPT 023/11 e NBR 10897/2014. Notas de aula, Curso de engenharia de segurança contra incêndio e pânico, Pontifícia Universidade Católica do Paraná, Curitiba 2018.

JÚNIOR, J. Sprinklers: conceitos básicos e dicas excelentes para profissionais um estudo prático sobre a NFPA 13. 1. Ed. Instituto sprinklers brasil, São Paulo, 2015.

NAKAMURA, Juliana; Sprinklers anti-incêndio: tubulação pode ser de aço carbono, tubos de cobre ou CPVC. Texto técnico. Disponível em: http://construcaomercado17.pini.com.br/negocios-incorporacaoconstrucao/161/sprinklers-anti-incendio-tubulacao-pode-ser-de-aco-carbono-tubos-de333896-1.aspx. Acesso em: 01 de junho de 2018.

PANNONI. F. Princípios da galvanização a fogo. 2015. Disponível em: $<$ https://www.researchgate.net/publication/266345339_Principios_da_Galvanizacao_a _Fogo> Acesso em 16 de junho de 2018.

PEREIRA, A. ARAUJO,C. Sistema de chuveiros automáticos. Revista Científica Aprender.4ed. Jun, 2011. Disponível em: $<$ http://revista.fundacaoaprender.org.br/index.php?id=141>. Acesso em: 10 de junho de 2018.

SKOP. Sprinklers: o guia essencial. E-book, 2017. Disponível em:<http://www.skop.com.br/2018/05/29/sprinklers-o-guia-essenciall> Acesso em: 25 de maio de 2018.

TELLES. P. Tubulações industriais materiais, projeto, montagem. 10. Ed. LTC, Rio de Janeiro, 2001.

TRINDADE, P. Meios de extinção de incêndio sistemas automáticos por água. 2009. Dissertação (Mestrado em engenharia civil) - Faculdade de engenharia faculdade do Porto - FEUP, Porto, Portugal, 2009.

TUPY S.A. Catálogo Técnico: CT1003. Joinville, 2009 Disponível em: $<$ http://www.tupy.com.br/downloads/pdfs/conexoes/catalogo_pt.pdf $>$. Acesso em: 01 de junho de 2018. 